\title{
Portal Fibroblasts in Biliary Fibrosis
}

\author{
Rebecca G. Wells
}

Published online: 14 September 2014

(C) Springer Science+Business Media New York 2014

\begin{abstract}
Portal fibroblasts are a minor population in the normal liver, found in the periportal mesenchyme surrounding the bile ducts. While many researchers have hypothesized that they are an important myofibroblast precursor population in biliary fibrosis, responsible for matrix deposition in early fibrosis and for recruiting hepatic stellate cells, the role of portal fibroblasts relative to hepatic stellate cells is controversial. Several papers published in the past year have addressed this point and have identified other potential roles for portal fibroblasts in biliary fibrosis. The goal of this review is to critically assess these recent studies, to highlight gaps in our knowledge of portal fibroblasts, and to suggest directions for future research.
\end{abstract}

Keywords Desmin - Elastin · Mesothelin - Collagen XV · Lecithin-retinol acyltransferase $\cdot$ Bile duct ligation

\section{Introduction}

Fibrosis is often referred to as the "final common pathway" occurring after liver injury. The location and etiology of liver injury, however, have pronounced effects on the nature and mechanism of fibrosis. This is particularly true in biliary fibrosis, also known as cholestatic fibrosis, such as occurs in primary sclerosing cholangitis, primary biliary cirrhosis, and biliary atresia. Biliary fibrosis differs from other forms of liver fibrosis in significant ways: it is often

\section{R. G. Wells ( $\square)$}

Department of Medicine, Perelman School of Medicine, University of Pennsylvania, 905 BRB II/III, 421 Curie Blvd, Philadelphia, PA 19104, USA

e-mail: rgwells@mail.med.upenn.edu more rapid, involves dysregulated proliferation of bile ducts and is associated with cholestasis, and is localized, at least initially, to the periductal region. Most important, biliary fibrosis results from injury to cholangiocytes, rather than to hepatocytes, and these cholangiocytes are active participants in the process $[1,2]$. What is still unclear, however, is whether biliary fibrosis requires specialized fibrogenic myofibroblasts derived not from hepatic stellate cells but from portal fibroblasts. This controversy and the potential roles-fibrogenic and otherwise-of portal fibroblasts in biliary fibrosis are the subject of this review.

Over the last decade four major categories of cells have been identified as potential precursors of the fibrogenic myofibroblasts that drive liver fibrosis: cholangiocytes and hepatocytes which undergo an epithelial to mesenchymal transition (EMT), fibrocytes from the bone marrow, portal and other fibroblasts, and hepatic stellate cells [3-5]. Recent research suggests that, at least in rodent models, fibrocytes contribute only a small number of cells to the myofibroblast population [6, 7], and epithelial cells undergoing EMT virtually none [8-10]; most authors agree that hepatic stellate cells are the predominant myofibroblast population in fibrosis resulting from hepatocyte injury. In spite of elegant work from several groups, however, the relative contributions of hepatic stellate cells and portal fibroblasts in biliary fibrosis remain uncertain. Specifically, it is unclear whether portal fibroblasts yield a major myofibroblast population and, if yes, whether that contribution is primarily at early time points. Additionally, research has not yet established whether portal fibroblasts in vivo deposit the fibrillar collagens typical of fibrosis or whether they have other roles in fibrosis.

Portal fibroblasts are a heterogeneous group of fibroblasts found in the mesenchyme of the portal tract, surrounding the intrahepatic bile ducts. (Note that the cells responsible for fibrosis of the extrahepatic bile ducts are 
not known. This discussion concerns only fibrosis occurring in the liver properly, although it includes intrahepatic biliary fibrosis resulting from extrahepatic ductal fibrosis and obstruction.) In comparison to hepatic stellate cells, which were first visualized (and beautifully sketched) a century and a half ago by Carl Wilhelm von Kupffer [11], fibroblasts in the portal tract were, to our knowledge, first illustrated in the literature in 1961 [12] and received significant attention as potential mediators of biliary fibrosis relatively recently [13-16]. While lineage tracing studies show that portal fibroblasts and hepatic stellate cells (as well as smooth muscle cells and fibroblasts located around the central veins) are derived from a common Wilms tumor 1-expressing precursor lineage in the mesenchyme of the septum transversum [17], they are distinct cell populations in the adult animal, with different localizations (portal mesenchyme vs. space of Disse), different functions [18], and different marker expressions. Hepatic stellate cells are commonly identified by the presence of vitamin A-containing lipid droplets or by the expression of a variety of markers including desmin, cytoglobin, glial fibrillary acidic protein (GFAP), cellular retinol binding protein 1, and Hand2, although the usefulness of these markers varies with the species and the degree of myofibroblastic activation $[19,20]$. Two recent studies, both carefully validated, identified the PDGF receptor $\beta$ subunit and lecithin-retinol acyltransferase (LRAT) as new markers that specifically label mouse hepatic stellate cells, regardless of activation state $[21 \bullet \cdot, 22 \bullet \cdot]$; these will likely see widespread use in the future. Portal fibroblasts have been identified by a variety of markers including Thy1, fibulin-2, elastin, IL-6, cofilin1 , and the ectonucleotidase NTPDase 2 [19, 23]. Two groups recently compared the transcriptomes of portal fibroblasts and hepatic stellate cells in order to identify new portal fibroblast markers. One group identified COL15A1 [24••], while the other found calcitonin $\alpha$ and mesothelin (along with a long list of other genes) to be specific portal fibroblast markers [25••]. All of these new portal fibroblast markers require validation.

\section{The Relative Contributions of Hepatic Stellate Cells and Portal Fibroblasts in Matrix Deposition in fibrosis}

\section{Studies Showing that Portal Fibroblasts are a Major Myofibroblast Population in Fibrosis}

There are conflicting data in the literature about the role of portal fibroblasts in matrix deposition in biliary fibrosis. While the ability of portal fibroblasts to differentiate into fibrogenic, $\alpha$-smooth muscle actin (SMA)-expressing myofibroblasts in vitro has been well documented, their role in vivo is less certain. In favor of portal fibroblasts being major players in biliary fibrosis, many groups have used marker analyses to argue that there is clear in vivo evidence that there is a significant population of fibrogenic myofibroblasts in biliary fibrosis not derived from hepatic stellate cells. Given the controversy in this area, it is important to examine carefully the methods used in these studies.

One of the first papers implicating portal fibroblasts in biliary fibrosis was published in 1996 by Tuchweber et al. [16]. This group carried out bile duct ligations in rats, examining time points from 1 to 7 days post procedure. Using double immunostaining techniques and defining portal fibroblast-derived myofibroblasts as $\alpha$-SMA-positive, desmin-negative cells, and hepatic stellate cellderived myofibroblasts as $\alpha$-SMA-positive, desmin-positive cells, these investigators concluded that myofibroblasts were mostly derived from portal fibroblasts in the first $72 \mathrm{~h}$ after injury, and from hepatic stellate cells thereafter.

A decade later, Beaussier et al. induced biliary injury in rats by two methods, bile duct ligation and arterial ischemia [26]. They found that prominent fibrosis developed, with the appearance of many $\alpha$-SMA-positive myofibroblasts; few of these, however, were in the same region as desmin-expressing cells, leading the authors to conclude that most myofibroblasts in the portal region were derived from portal mesenchymal cells such as portal fibroblasts, and not from hepatic stellate cells. Note, however, that double staining was not done. Like the work by Tuchweber et al., this study relied on desmin staining to identify hepatic stellate cell-derived myofibroblasts, and defined portal fibroblasts by the absence rather than the presence of a specific marker. Whether immunostaining for desmin was sufficient in either study to identify all hepatic stellate cells is unclear; some desmin-negative cells express the PDGF receptor $\beta$ subunit [14], which has since been found to be specific for hepatic stellate cells [21••]. Thus, the strength of the conclusions in these two influential studies is dependent on the efficiency of desmin immunostaining and on the validity of desmin as a sensitive and specific stellate cell marker in vivo, and is therefore difficult to assess.

In a recently published study, Iwaisako et al. used transgenic mice expressing green fluorescent protein (GFP) under the control of the collagen $\alpha 1$ (I) promoter (such that all collagen-expressing cells are green) to support the contention that portal fibroblasts play a prominent role in biliary fibrosis [25••]. The authors identified hepatic stellate cells by the presence of vitamin A, arguing in this and a previous paper that all stellate cells, even after myofibroblastic differentiation, contain at least small amounts of vitamin A [25••, 27•]. Portal fibroblasts and myofibroblasts were defined as vitamin A-negative cells in the nonparenchymal cell fraction after liver digestion. Control experiments making use of other known hepatic stellate 
cell and portal fibroblast markers (desmin and GFAP vs. Thy 1 and elastin, respectively) suggested that this approach effectively segregated the two cell types. The authors found a significant percentage of vitamin A-negative, GFP-positive cells in all forms of fibrosis, including after hepatocyte injury from chronic carbon tetrachloride $\left(\mathrm{CCl}_{4}\right)$ intoxication. Remarkably, they observed that an average of $73 \%$ of myofibroblasts in livers 5 days after bile duct ligation was vitamin A negative and therefore not stellate cell derived; this decreased with time but still remained significant at $46 \%$ on day 20 after bile duct ligation. Only a small percentage of these cells $(<4 \%)$ expressed fibrocyte markers, suggesting that portal fibroblasts give rise to the dominant myofibroblast population in biliary fibrosis, particularly at early time points.

Iwaisako et al. also carried out gene expression analyses comparing stellate cells and portal fibroblasts after different forms of injury. Surprisingly, the gene profiles of stellate cells and portal fibroblasts from bile duct-ligated animals had more in common than did the gene profiles of stellate cells from bile duct-ligated versus $\mathrm{CCl}_{4}$-treated animals [25••]. This raises the possibility that the mechanism of injury has as much to do with myofibroblast function as does the cell of origin. These authors also found that the level of Collal mRNA expression in a given myofibroblast population was directly related to the prominence of that population at a given time [25••]; in other words, portal myofibroblasts are particularly fibrogenic at early time points after biliary injury when they comprise the bulk of the myofibroblast population.

These findings are consistent with portal fibroblasts serving as "first responders" after biliary injury, as proposed in 2002 by Kinnman and Housset [28]. Iwaisako et al. suggest a potential mechanism whereby portal myofibroblasts could mediate the first wave of collagen deposition but recruit and ultimately be supplanted by hepatic stellate cells. They show that portal myofibroblasts express IL-25R, enabling them to respond to IL-25, which is upregulated in the serum after bile duct ligation [25••]; IL25-treated portal myofibroblasts secrete IL-13, which increases hepatic stellate cell activation and fibrogenesis.

\section{Studies Showing that Portal Fibroblasts are a Minor} Myofibroblast Population in Fibrosis

At odds with the data reviewed above is a recently published, methodologically sophisticated lineage tracing paper showing that portal fibroblasts are at best minor contributors to biliary fibrosis [22••]. Mederacke et al. generated transgenic mice with a bacterial artificial chromosome expressing Cre recombinase under the control of the lecithin-retinol acyltransferase (Lrat) gene; this resulted in highly efficient and specific labeling of hepatic stellate cells, as defined by expression of desmin and the PDGF receptor $\beta$ subunit $(P d g f r b)$ and by the presence of vitamin A-containing lipid droplets. There was no cross-labeling of portal fibroblasts or other cells of the liver (excepting a small number of vascular smooth muscle cells) after crossing to a reporter mouse. LRAT drives the formation of lipid droplets containing retinyl esters, and it is thus reasonable that its expression is specific to hepatic stellate cells.

Mederacke et al. tested a total of 7 fibrosis models (hepatotoxic, biliary, and metabolic) using these transgenic mice, demonstrating that 82-96\% of myofibroblasts are derived from hepatic stellate cells, even in the early stages of biliary fibrosis. They also isolated a small number of Lrat-negative portal myofibroblast-like cells but found that they secreted less collagen than hepatic stellate cell-derived myofibroblasts. Thus, these authors concluded that the majority of myofibroblasts, and the dominant population of collagenproducing cells in both biliary and non-biliary fibrosis, consisted of hepatic stellate cells, with portal fibroblast-like cells playing a minor role in matrix deposition.

\section{Reconciling Conflicting Data on the Importance} of Portal Fibroblasts in Fibrosis

Reconciling these disparate pieces of data, particularly the work of Iwaisako et al. and Mederacke et al., is difficult. While Mederacke et al.

did observe a small number of portal myofibroblasts, the number of these cells was low compared to hepatic stellate cells, they were minimally fibrogenic, and there was no significant shift from portal fibroblast-derived to hepatic stellate cell-derived myofibroblasts as biliary fibrosis progressed-all findings in contrast to those of Iwaisako et al. One of the most significant problems in interpreting these studies is that portal fibroblasts are typically defined by the absence, rather than the presence, of specific markers. It will be key to validate newly identified markers for portal fibroblasts and to carry out portal fibroblast lineage tracing studies, following the model of Mederacke et al. Similarly, new stellate cell markers, including Lrat and Pdgfrb, need to be incorporated into studies similar to those of Iwaisako et al. An ideal, although technically daunting, approach would be to differentially label portal fibroblasts and hepatic stellate cells in the same mouse model, and thereby to directly compare their contributions in biliary and non-biliary models.

Henderson et al. developed a Pdgfrb-Cre mouse [21••] and demonstrated that, in the liver, they were able to label hepatic stellate cells (at all stages of differentiation) efficiently and specifically. This mouse, which was used to show that almost all myofibroblasts that appear after $\mathrm{CCl}_{4}$ treatment are derived from hepatic stellate cells, would be 
useful for validating the experiments of Mederacke et al. [22••], especially in biliary fibrosis. Additionally, the approach of Puche et al. would be valuable if applied specifically to portal fibroblasts and hepatic stellate cells in biliary fibrosis models [29•]. Puche et al. expressed the herpes simplex virus thymidine kinase under the control of the GFAP promoter; expression of the viral gene led to death of cells in which the gene was expressed. These authors considered GFAP to be specific for hepatic stellate cells, although others have shown it to be expressed in other cells including portal fibroblasts [22••, 23]; use of this method in combination with new and highly specific stellate cell and portal fibroblast promoters would enable a direct determination of the functional relevance of these two cell types in various models of murine liver fibrosis.

A related issue (but one that could be studied with similar techniques) is the fate of portal fibroblasts and myofibroblasts after fibrosis regression. Two groups recently demonstrated independently and using different methods that, while about half of myofibroblastic hepatic stellate cells undergo apoptosis, the remaining cells revert to an intermediate, "primed" state [27, 30•]. Whether the same is true for portal fibroblasts during regression of biliary fibrosis (or, potentially, after the initial phases of biliary fibrosis) requires study, as it has significant implications for hepatic stellate cell function, cholangiocyte proliferation and, potentially, bile duct integrity (see below).

\section{Potential Roles for Portal Fibroblasts in Biliary Fibrosis Beyond Fibrogenesis}

\section{Portal Fibroblasts Regulate Cholangiocyte Behavior}

Portal fibroblasts have potential roles in biliary fibrosis independent of matrix deposition, including crosstalk with cholangiocytes. Jhandier et al. proposed a model whereby portal myofibroblasts regulate bile duct proliferation [31]. These authors showed that portal fibroblasts in the normal liver express the ectonucleotidase NTPDase 2 (which is used as a marker of these cells). This enzyme hydrolyzes extracellular nucleotides which would otherwise bind to P2Y family receptors on cholangiocytes and stimulate cholangiocyte proliferation. Thus, portal fibroblasts in the normal liver prevent, or at least fail to stimulate, ductular expansion. Portal myofibroblasts, however, do not express NTPDase 2, such that in the diseased state, extracellular nucleotides are theoretically available to enhance bile duct proliferation [3, 31].

Portal fibroblasts may also regulate cholangiocyte function via a peribiliary stem cell niche, or by deposition of hyaluronic acid or other matrix proteins during development or after injury [32-36]. Additional studies examining reciprocal expression of growth factors and their receptors by cholangiocytes and portal fibroblasts (and the impact of these interactions on hepatic stellate cells), similar to those carried out by Iwaisako et al. (above), will be important to understanding the full role of portal fibroblasts in biliary injury [1, 37].

Portal Fibroblasts may Regulate Angiogenesis and Maintain Bile Duct Integrity in Fibrosis

It is possible that the major function of portal fibroblasts in cholestatic fibrosis is unrelated to collagen deposition or to myofibroblasts. We have previously suggested that portal fibroblasts should be studied in more detail with regard to classical fibroblast functions, including site-specific matrix protein deposition, interactions with the epithelium, and expression of cell migration guidance signals [38]. There is evidence supporting important roles for portal fibroblasts in at least the first two categories [38].

Additional functions are worth considering. A recent paper from Lemoinne et al. puts forth the novel hypothesis that portal myofibroblasts are a minor population of myofibroblasts that nonetheless play a key role in angiogenesis associated with fibrosis and cirrhosis [24••]. This group compared the gene expression profiles of activated hepatic stellate cells and portal fibroblasts in culture, determining that expression of the basement membrane collagen component Coll $15 \mathrm{al}$ was specific to portal myofibroblasts in the liver. Cells expressing this marker increased dramatically in advanced fibrosis and cirrhosis, and were closely apposed to vascular structures in fibrotic septae and around reactive ductules. In vitro, these cells were stimulated by cholangiocytes to produce VEGFAcontaining microparticles, which markedly upregulated tubulogenesis and angiogenesis in several assays [24••]. Validation of Col15al as a portal fibroblast marker is required, as there are additional studies to confirm that these cells are the same population of portal myofibroblasts studied by other groups. Nonetheless, this work suggests new roles for portal fibroblasts in fibrosis and new modes of interacting with cholangiocytes, particularly in established cirrhosis. Note that most of the other studies discussed in this review have viewed portal fibroblasts as potential early responders after injury and none, including those from Mederacke et al. and Iwaisako et al., used animal models of established cirrhosis; these models will be critical to developing a full understanding of portal fibroblast function.

Portal fibroblasts possibly maintain ductal structure and integrity. Unlike hepatic stellate cells, portal fibroblasts deposit elastin in addition to fibrillin (both components of elastic fibers), particularly after bile duct ligation [39]; elastic fibers may enhance the resilience of the duct in the setting of obstruction, where there is distension and increased ductal pressure. It may be relevant that portal myofibroblasts secrete 
collagen in addition to elastin, since this combination could enhance the structural stability of the duct. Collagen XV, which was recently identified as a portal fibroblast marker, is known primarily as a structural collagen that underlies blood vessels and maintains basement membrane integrity [24••, 40, 41]. It has yet not been well studied in liver, but could also conceivably provide mechanical benefits to the bile ducts.

\section{Conclusions}

Portal fibroblasts were first described more than 50 years ago, yet in spite of rigorous studies published in the last year, their function in the normal and fibrotic liver remains controversial. Confusion stems in part from the heterogeneous and, to some extent, poorly defined nature of this population. The recent identification of new, highly specific markers for both stellate cells and portal fibroblasts should lead to the development of new tools to study the behavior of both cell types in more depth. Portal fibroblasts may have unexpected functions, including roles in angiogenesis and bile duct structure, and thus future research on portal fibroblasts in biliary fibrosis should include not only early fibrosis models but also those with established fibrosis and significant angiogenesis. The fate of portal myofibroblasts as fibrosis progresses and regresses should also be part of these studies. Finally, while initial studies must include lineage tracing analyses in mice, it will ultimately be important to extend this work to human materials.

Acknowledgments Funded in part by NIH R01 DK-058123 to Rebecca G. Wells.

\section{Compliance with Ethics Guidelines}

Conflict of Interest Rebecca G. Wells declares no conflicts of interest.

Human and Animal Rights and Informed Consent This article does not report any studies with human or animal subjects.

\section{References}

Papers of particular interest, published recently, have been highlighted as:

- Of importance

-• Of major importance

1. Fabris L, Strazzabosco M (2011) Epithelial-mesenchymal interactions in biliary diseases. Semin Liver Dis 31(1):11-32

2. O'Hara SP et al (2013) The dynamic biliary epithelia: molecules, pathways, and disease. J Hepatology 58(3):575-582
3. Fausther M, Lavoie EG, Dranoff JA (2013) Contribution of Myofibroblasts of Different Origins to Liver Fibrosis. Current Pathobiology Reports 1(3):225-230

4. Friedman SL (2008) Mechanisms of hepatic fibrogenesis. Gastroenterology 134(6):1655-1669

5. Novo E et al (2009) Hepatic myofibroblasts: a heterogeneous population of multifunctional cells in liver fibrogenesis. Int $\mathbf{J}$ Biochem Cell Biol 41(11):2089-2093

6. Kisseleva $\mathrm{T}$ et al (2006) Bone marrow-derived fibrocytes participate in pathogenesis of liver fibrosis. J Hepatology 45(3):429-438

7. Scholten D et al (2011) Migration of fibrocytes in fibrogenic liver injury. Am J Path 179(1):189-198

8. Chu AS et al (2011) Lineage tracing demonstrates no evidence of cholangiocyte epithelial-to-mesenchymal transition in murine models of hepatic fibrosis. Hepatology 53(5):1685-1695

9. Scholten D et al (2010) Genetic labeling does not detect epithelial-to-mesenchymal transition of cholangiocytes in liver fibrosis in mice. Gastroenterology 139(3):987-998

10. Taura $\mathrm{K}$ et al (2010) Hepatocytes do not undergo epithelialmesenchymal transition in liver fibrosis in mice. Hepatology 51(3):1027-1036

11. Haubrich WS (2004) Kupffer of Kupffer cells. Gastroenterology 127(1): 16

12. Steiner JW, Carruthers JS (1961) Studies on the fine structure of proliferated bile ductules. II. Changes of the ductule-connective tissue envelope relationship. Can Med Assoc J 85:1275-1287

13. Cassiman D et al (2002) Hepatic stellate cell/myofibroblast subpopulations in fibrotic human and rat livers. J Hep 36(2):200-209

14. Kinnman $\mathrm{N}$ et al (2003) Myofibroblastic conversion of peribiliary fibrogenic cells distinct from hepatic stellate cells is stimulated by platelet-derived growth factor during liver fibrogenesis. Lab Invest 83(2): 163-173

15. Knittel T et al (1999) Localization of liver myofibroblasts and hepatic stellate cells in normal and diseased rat livers: distinct roles of (myo-)fibroblast subpopulations in hepatic tissue repair. Histochem Cell Biol 112(5):387-401

16. Tuchweber B et al (1996) Proliferation and phenotypic modulation of portal fibroblasts in the early stages of cholestatic fibrosis in the rat. Lab Invest 74(1):265-278

17. Asahina $\mathrm{K}$ et al (2011) Septum transversum-derived mesothelium gives rise to hepatic stellate cells and perivascular mesenchymal cells in developing mouse liver. Hepatology 53(3):983-995

18. Friedman SL (2008) Hepatic stellate cells: protean, multifunctional, and enigmatic cells of the liver. Physiol Rev 88(1):125-172

19. Lemoinne $\mathrm{S}$ et al (2013) Origins and functions of liver myofibroblasts. Biochim Biophys Acta 1832(7):948-954

20. Yin $\mathrm{C}$ et al (2012) The basic helix-loop-helix transcription factor, heart and neural crest derivatives expressed transcript 2, marks hepatic stellate cells in zebrafish: analysis of stellate cell entry into the developing liver. Hepatology 56(5):1958-1970

21. • Henderson NC et al (2013) Targeting of alphav integrin identifies a core molecular pathway that regulates fibrosis in several organs. Nat Med 19(12):1617-1624 Although not its major goal, this study established Pdgfrb as a specific marker of both quiescent and activated hepatic stellate cells, and showed that hepatic stellate cell-derived myofibroblasts are the major myofibroblasts in $\mathrm{CCl}_{4}$-induced fibrosis

22. • Mederacke I et al (2013) Fate tracing reveals hepatic stellate cells as dominant contributors to liver fibrosis independent of its aetiology. Nat Commun 4:2823. This elegant lineage tracing study established Lrat as a specific marker of both quiescent and activated hepatic stellate cells and showed in multiple mouse 
models of fibrosis that hepatic stellate cells give rise to the major myofibroblast population at both early and late time points

23. Li Z et al (2007) Transforming growth factor-beta and substrate stiffness regulate portal fibroblast activation in culture. Hepatology 46(4):1246-1256

24. •• Lemoinne S et al (2014) Portal myofibroblasts promote vascular remodeling underlying cirrhosis formation through the release of microparticles. Hepatology Epub 2014/07/22. This study identified the basement membrane collagen component Coll5al as a specific marker of portal myofibroblasts and showed that these cells play a key role in angiogenesis associated with cirrhosis

25. • Iwaisako K et al (2014) Origin of myofibroblasts in the fibrotic liver in mice. Proc Nat Acad Sci U S A Epub 2014/07/31. This study identified mesothelin and other portal fibroblast specific markers and demonstrated that portal fibroblasts give rise to the dominant, highly fibrogenic myofibroblast population in biliary fibrosis, particularly at early time points

26. Beaussier M et al (2007) Prominent contribution of portal mesenchymal cells to liver fibrosis in ischemic and obstructive cholestatic injuries. Lab Invest 87(3):292-303

27. - Kisseleva T et al (2012) Myofibroblasts revert to an inactive phenotype during regression of liver fibrosis. Proc Natl Acad Sci U S A 109(24):9448-9453. One of two studies demonstrating that, during fibrosis regression, approximately half of stellate cell myofibroblasts undergo apoptosis while the other half revert to an intermediate state, primed for rapid reactivation after repeat injury

28. Kinnman N, Housset C (2002) Peribiliary myofibroblasts in biliary type liver fibrosis. Front Biosci 7:d496-d503

29. - Puche JE (2013) A novel murine model to deplete hepatic stellate cells uncovers their role in amplifying liver damage in mice. Hepatology 57(1):339-350. This study depleted hepatic stellate cells and demonstrated that liver damage as well as fibrosis was decreased in multiple models, including bile duct ligation

30. - Troeger JS et al (2012) Deactivation of hepatic stellate cells during liver fibrosis resolution in mice. Gastroenterology
143(4):1073-1083 e22. One of two studies demonstrating that, during fibrosis regression, approximately half of stellate cell myofibroblasts undergo apoptosis while the other half revert to an intermediate state, primed for rapid reactivation after repeat injury

31. Jhandier MN et al (2005) Portal fibroblasts regulate the proliferation of bile duct epithelia via expression of NTPDase2. J Biol Chem 280(24):22986-22992

32. He $\mathrm{Y}$ et al (2008) Interaction of CD44 and hyaluronic acid enhances biliary epithelial proliferation in cholestatic livers. Am J Physiol Gastrointest Liver Physiol 295(2):G305-G312

33. Boulter L et al (2012) Macrophage-derived Wnt opposes Notch signaling to specify hepatic progenitor cell fate in chronic liver disease. Nat Med 18(4):572-579

34. Greenbaum LE, Wells RG (2011) The role of stem cells in liver repair and fibrosis. Int J Biochem Cell Bio 43(2):222-229

35. Omenetti A et al (2007) Hedgehog-mediated mesenchymal-epithelial interactions modulate hepatic response to bile duct ligation. Lab Invest 87(5):499-514

36. Van Hul NK et al (2009) Relation between liver progenitor cell expansion and extracellular matrix deposition in a CDE-induced murine model of chronic liver injury. Hepatology 49(5):1625-1635

37. Dranoff JA, Wells RG (2010) Portal fibroblasts: Underappreciated mediators of biliary fibrosis. Hepatology 51(4):1438-1444

38. Wells RG (2014) The portal fibroblast: not just a poor man's stellate cell. Gastroenterology 147(1):41-47

39. Guyot C, Combe C, Desmouliere A (2006) The common bile duct ligation in rat: A relevant in vivo model to study the role of mechanical stress on cell and matrix behaviour. Histochem Cell Biol 126(4):517-523

40. Rygh CB et al (2014) Image-based assessment of microvascular function and structure in collagen XV- and XVIII-deficient mice. J Physol 592(Pt 2):325-336

41. Clementz AG et al (2013) Collagen XV inhibits epithelial to mesenchymal transition in pancreatic adenocarcinoma cells. PLoS ONE 8(8):e72250 